\title{
The Impact of Heterogeneity and Order in Heavy Duty Vehicle Platooning Networks (Poster)
}

\author{
Kuo-Yun Liang \\ Scania CV AB \\ 15187 Södertälje, Sweden \\ Email: kuo-yun.liang@ @scania.com
}

\author{
Assad Alam \\ Scania CV AB \\ 15187 Södertälje, Sweden \\ Email: alam.assad@scania.com
}

\author{
Ather Gattami \\ KTH - Royal Institute of Technology \\ 10044 Stockholm, Sweden \\ Email: gattami@kth.se
}

\begin{abstract}
It is formally known that by establishing a heavy duty vehicle platoon, the fuel consumption is reduced for the follower vehicle due to the lower air drag. However, it is not clear how the platoon should be formed with respect to the heavy duty vehicle properties. String stability is a well discussed issue in vehicle platooning. However, each vehicle's properties have to be taken into consideration when analyzing the platoon system. In this paper, we analyze one property of heavy duty vehicles - the mass. The results show that the robustness is influenced by the order and physical characteristics of the vehicles in the platoon. When utilizing identical PID controllers for all vehicles in the platoon, it is better to arrange the heaviest vehicle first with decreasing mass order when considering the platoon behavior. However, in reality it is difficult to start rearranging a platoon in the middle of a highway and it would also require $\mathrm{V} 2 \mathrm{~V}$ communication. A controller is often optimized for a particular configuration set that can cause slinky effects to the platoon. Therefore, a mass-dependent PID controller is introduced to establish a better platoon behavior for heavy duty vehicles. The results show no slinky effects regardless of the vehicle order in the platoon.
\end{abstract}

\section{INTRODUCTION}

Vehicle platooning, also known as a convoy, has become a popular topic for the vehicle industry due to the increased traffic intensity. A platoon can be formed with commercial systems that exist today such as the adaptive cruise control (ACC), which is an extension to the cruise control (CC). The $\mathrm{CC}$ is a system that automatically maintains the vehicle speed according to a speed reference set by the driver. The ACC adapts the vehicle speed, if the speed of the vehicle ahead is lower, by utilizing radar that measures relative distance and relative speed. The ACC allows the driver to set a given time headway distance between its own and the vehicle ahead. By decreasing the intermediate distance between each vehicle on a highway, the traffic flow is increased. Furthermore, by establishing vehicle-to-vehicle (V2V) communication the intermediate distance can be decreased further due to more information can be transmitted, which allows the platoon network to act more as one single unit. Also, if a heavy duty vehicle (HDV) platoon is formed, the emission can be reduced significantly due to the lower wind resistance that the follower vehicle experiences. Studies in [1] showed that a fuel reduction of $4.7 \%-7.7 \%$ can be obtained with two identical HDVs traveling closely on a highway.

Early work on vehicle platoons and control, where the issue of string stability were raised, were presented in [2], [3] and [4]. Further studies regarding string stability were conducted in [5], [6] and [7]. The models, in these results, describes each vehicle in a homogeneous platoon as a simple linear system to guarantee string stability. The effects of heterogeneity on the stability margin was studied in [8]. The study utilized a bidirectional control law on a double integrating agent system with a partial differential equation approximation to achieve the results. It is essential to analyze string stability for a heterogeneous platoon for real time applications.

In this work, we focus on analyzing how variations in the vehicle mass can affect the robustness of a platoon. The HDV mass can vary during a transportation assignment due to loading and unloading goods. This change of the vehicle gross weight can affect the vehicle dynamics. Each vehicle is assumed to utilize identical proportional-integral-derivative (PID) controller [9]. The HDV platooning system that we consider resembles a mass-spring system with identical springs. If the masses are different, a damping effect is introduced into the system. Now consider a scenario with several identically configured HDVs but with varying masses. The HDVs, being of the same model and from the same manufacturer, have identical ACC systems that govern the speed of each vehicle in the platoon. It is most likely that all the HDVs will behave differently during acceleration and braking due to the different masses. Also, the platoon would behave differently depending on how the HDVs were arranged. We suggest a solution that does not require any rearrangement of the platoon or requires to alter the ACC logic, but only to tweak the controller slightly to enhance the platoon behavior. This work deviates from the past works by introducing a damping effect to the system for a more realistic application, with exception to [6], and by analyzing the transfer function of the relative distance between the vehicles.

Hence, the main contribution of this paper is to investigate how different vehicle masses contributes to the string stability criteria when utilizing identical PID controllers. The controller is purposely configured such that it does not fulfill the string stability criteria for a set of masses. Therefore the aim of this work is not to investigate the optimal PID controller for platooning, but to introduce a general modified PID controller that can contribute for a better platoon behavior.

The outline of this paper is as follows. First we give 


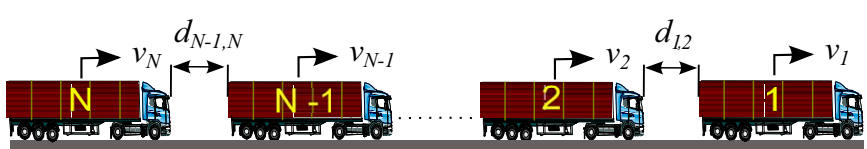

Fig. 1. Vehicle platooning of $\mathrm{N}$ vehicles where the lead vehicle is denoted as $i=1$ and the tail end vehicle as $i=N$. The speed of the $i$ :th vehicle is denoted as $v_{i}$ and the intermediate distance between vehicle $i$ and $i-1$ is denoted as $d_{i-1, i}$.

a general description of string stability and its criteria in Section II. The problem formulation is presented in Section III. In Section IV the system model is presented together with the analysis and simulations of how the mass affects string stability. Due to the inconvenience of rearranging a platoon in a real time scenario, Section $\mathrm{V}$ suggests a slightly modified PID controller that regards the vehicle mass for a more robust platoon behavior. Finally, in Section VI we present a brief summary of the results in this paper and conclusions.

\section{String Stability}

The nomenclature, used here, for vehicle platooning is defined as shown in Fig. 1. The platoon consists of $i=$ $\{1,2, \ldots, N\}$ vehicles, where $i=1$ is the lead vehicle of the platoon and $i=N$ is the tail end vehicle. $v_{i}$ denotes the velocity of the $i$ :th vehicle and $d_{i-1, i}$ denotes the intermediate distance between the $i$ th vehicle and the preceding vehicle.

In a platoon, a change in velocity can be amplified and propagated throughout the platoon. The disturbance could lead to an unwanted platoon behavior, also known as the slinky effect, which may result in vehicle collisions. Concerns regarding the robustness of the platoon have often been raised. Thus, it is of most importance to prevent this from occurring. In [10], a rigorous definition of string stability is presented. String stability can be seen as a robustness analysis for vehicle platooning that can briefly be described as the ability to subdue disturbances throughout the platoon and prevent slinky effects. A slightly less strict formulation of string stability, presented in [11] and [12], will be used instead.

Assuming that the headway distance $d_{i-1, i}$ between vehicle $i$ and $i-1$ is controlled by vehicle $i$ with only information regarding the vehicle ahead, the relation between both vehicles can be expressed as:

$$
v_{i}(s)=G_{i}^{v}(s) v_{i-1}(s)
$$

where $G_{i}^{v}(s)$ is the velocity transfer function from the $i-1$ :th to the $i$ :th vehicle and $v_{i}(s)$ is the Laplace transform of the velocity $v_{i}(t)$.

In order for a platoon of $\mathrm{N}$ vehicle to be string stable, the following is defined:

$$
\left\|G_{i}^{v}(s)\right\|_{\infty} \leq 1
$$

for all $i \in[2, N]$, where $\|\cdot\|_{\infty}$ is the maximum peak of the transfer function gain, which states that a disturbance in the velocity of a preceding vehicle will not be amplified downstream.

\section{PROBLEM DESCRIPTION}

We consider a simple linear time-invariant (LTI) vehicle model given as,

$$
m a+b v=u
$$

where $\mathrm{m}$ denotes the vehicle mass, $\mathrm{a}$ is the acceleration, $\mathrm{b}$ is commonly referred to as the air drag coefficient, $v$ denotes the velocity and $\mathrm{u}$ is the propulsion force. The force can then be interpreted as an input signal from the ACC controller, which can be approximated as a PID controller, $u=P I D$, where the PID controller utilizes the information of velocity and relative distance of the preceding vehicle. By expanding this to several vehicles, the whole system can be described as a platoon.

Now consider the simplified system of a HDV platoon consisting of $\mathrm{N}$ vehicles, where each vehicle characteristics differ only in mass and the vehicles utilize identical PID controllers. Every vehicle only receives information regarding intermediate distance and velocity of the vehicle ahead.

The objective is to obtain a better understanding on how the different masses affects the robustness of the platoon and if it is possible to obtain a string stable platoon by rearranging the order of the vehicles in the platoon for a specific controller configuration. This can be compared to a real scenario with identical HDVs, besides the mass, from the same manufacturer utilizing a commercial ACC. Even though a rearrangement of the platoon order may enhance the platoon behavior, it is inconvenient to do it in practical. Therefore, the aim is also to find a solution or suggestion to solve this problem without adding any further complexity to the system.

\section{ANALYSIS OF MASS VARIATIONS}

In this section, we present the system model and the derivation for the transfer functions of velocity and relative distance in order to analyze how the property of mass variation affects the robustness of the platoon. Furthermore, simulations are done to ensure the theory.

\section{A. System model}

As presented in [13], a vehicle platoon can be simplified as a mass-spring-damper system. To simplify calculations but yet keep the essential behavior, we express each vehicle as:

$$
m_{i} \ddot{x}_{i}+b_{i} \dot{x}_{i}=u_{i}
$$

where the index $i$ denotes the $i$ :th vehicle in the platoon, $m_{i}$ is the mass, $b_{i}$ is the damping effect that can also be seen as air drag resistance, $x_{i}$ is the longitudinal position, and $u_{i}$ is the longitudinal force of the $i$ :th vehicle. For simplicity of this study, the platoon is assumed to only travel on a flat road.

With the assumption that vehicle $i$ only has information of the vehicle ahead and that it is controlling the intermediate distance $d_{i-1, i}$, the following PID controller can be utilized:

$$
\begin{aligned}
u_{i}=P_{i}\left(d_{i-1, i}-\tau_{d} \dot{x}_{i}\right) & +I_{i} \int d_{i-1, i}-\tau_{d} \dot{x}_{i} \mathrm{~d} t \\
& +D_{i} \dot{d}_{i-1, i}
\end{aligned}
$$


where $d_{i-1, i}=x_{i-1}-x_{i}$ and $\tau_{d}$ denotes the desired time headway. One requirement is that $d_{i-1, i} \geq 0$ for all $i \in[2, N]$, which states whether the vehicles have collided.

All vehicles in the platoon are assumed to use identical controllers and have the same air drag resistance $b_{i}$. Even though identical controllers are used, the index $i$ in the controller will be kept to easier distinguish the controllers for the vehicles.

To analyze if the PID controller is robust according to the string stable criteria in (2), a transfer function for the system is obtained. By rewriting $d_{i-1, i}=x_{i-1}-x_{i}$ in the PID controller in (5) and inserting it into (4), the complete system for a HDV in a platoon is described. Hence, applying a Laplace transform on the system and obtaining the velocity of vehicle $i$ and $i-1$, the velocity transfer function $G_{i}^{v}(s)$ can then be expressed as:

$$
\begin{aligned}
G_{i}^{v}(s) & =\frac{s^{2} D_{i}+s P_{i}+I_{i}}{s^{3} m_{i}+s^{2}\left(b_{i}+\tau_{d} P_{i}+D_{i}\right)+s\left(P_{i}+\tau_{d} I_{i}\right)+I_{i}} \\
& =\frac{v_{i}(s)}{v_{i-1}(s)}
\end{aligned}
$$

Thus, if (6) fulfills the criteria in (2) for all $i \in[2, N]$, the platoon is considered to be string stable. Notice that the transfer function in (6) could also describe the relation between each vehicle in longitudinal position or acceleration.

It is obvious that the mass affects the maximum peak, but the main factor affecting the maximum peak is the choice of the PID controller, hence it is difficult to interpret how only the vehicle mass influence the robustness.

Another aspect that could be analyzed in platooning is the ability to suppress disturbances in relative distance. Although the string stability criteria concerns only position, velocity or acceleration differences between each vehicle, it could be as important to analyze how the relative distance propagates throughout the platoon. Relative distance between vehicles is a straightforward approach to study vehicle collisions. Similar to the velocity transfer function, by inserting (5) into (4) and apply the Laplace transform, the following transfer function is obtained:

$$
d_{i-1, i}(s)=\frac{s^{2} m_{i}+s\left(b_{i}+\tau_{d} P_{i}\right)+\tau_{d} I_{i}}{s^{2} D_{i}+s P_{i}+I_{i}} v_{i}(s)
$$

where $d_{i-1, i}(s)$ is the Laplace transform of the time domain relative distance $d_{i-1, i} \cdot v_{i}(s)$ can now be rewritten as $v_{i}(s)=$ $G_{i}^{v}(s) v_{i-1}(s)$ with $G_{i}^{v}(s)$ known from (6). This together with an index shift of (7) obtains the relative distance transfer function, which can be expressed as:

$$
G_{i}^{d}(s)=\frac{d_{i-1, i}}{d_{i-2, i-1}}=\frac{A_{i-1}(s)}{A_{i}(s)} \frac{B_{i-1}(s)}{C_{i}(s)}
$$

where

$$
\begin{aligned}
& A_{i}(s)=s^{2} m_{i}+s\left(b_{i}+\tau_{d} P_{i}\right)+\tau_{d} I_{i} \\
& B_{i}(s)=s^{2} D_{i}+s P_{i}+I_{i} \\
& C_{i}(s)=s^{3} m_{i}+s^{2}\left(b_{i}+\tau_{d} P_{i}+D_{i}\right)+s\left(P_{i}+\tau_{d} I_{i}\right)+I_{i}
\end{aligned}
$$

and the index $i$ denotes the $i$ :th vehicle in the platoon. Notice that the transfer function (8) consists of masses from two different vehicles. It is however rather difficult to interpret the platoon behavior from this function. The simple case is when both vehicle masses are identical, omitting the first quotient. This renders the relative distance transfer being the same as the velocity transfer function, $G_{i}^{d}(s)=G_{i}^{v}(s)$. However, with non-identical masses, a simplified transfer function is obtained by assuming the following:

$$
\left\{\begin{aligned}
\frac{P_{i-1}}{m_{i-1}} & \approx \frac{P_{i}}{m_{i}} \\
\frac{b_{i-1}}{m_{i-1}} & \approx \frac{b_{i}}{m_{i}} \\
\frac{I_{i-1}}{m_{i-1}} & \approx \frac{I_{i}}{m_{i}}
\end{aligned}\right.
$$

This is valid if the mass from the neighbor vehicle do not differ much. Hence, (8) can be approximated as:

$$
G_{i}^{d}(s) \approx \frac{m_{i}}{m_{i-1}} \frac{B_{i-1}(s)}{C_{i}(s)}
$$

where $B_{i-1}(s)$ and $C_{i}(s)$ are from (9). Similar to the string stability criteria in (2), we define that the relative distance transfer function should fulfill the same criteria, which states as $\left\|G_{i}^{d}(s)\right\|_{\infty} \leq 1$ for all $i \in[3, N]$, in order to subdue disturbances on the relative distance. It can immediately be seen that the mass quotient $\frac{m_{i}}{m_{i-1}}$ is a gain that directly affects the maximum peak value of $G_{i}^{d}(s)$. Thus, by arranging the platoon with a slightly heavier vehicle ahead all the time, the platoon should experience a better performance since the mass quotient gain will lower the maximum peak. Notice that only slightly heavier vehicle is placed ahead due to the assumptions in (10). This criteria cannot be guaranteed at all time in real scenario since a platoon mostly consists of vehicles with different masses in random order.

\section{B. Simulation}

To demonstrate the impact that the newly derived approximation $G_{i}^{d}(s)$ has on platooning, three different mass distributed platoons are studied. Small mass differences from each vehicle are used, due to the few assumptions made in the relative distance transfer function. The scenario consists of a ramp from standing still to $10 \mathrm{~m} / \mathrm{s}$ in $20 \mathrm{~s}$ using the velocity transfer function $G_{i}^{v}(s)$. This could be compared with a real scenario where the HDV platoon drives at approximately $50 \mathrm{~km} / \mathrm{h}$ on a road and enters a highway to a speed of $90 \mathrm{~km} / \mathrm{h}$. Due to the heavy vehicle, it takes approximately $20 \mathrm{~s}$ to gain that speed.

Each platoon consists of 81 vehicles, whereas each vehicle in the first platoon weight $40 \mathrm{t}$. The second platoon is arranged in mass increasing order, where the lead vehicle weights $20 \mathrm{t}$ and the tail vehicle weight $60 \mathrm{t}$. For simplicity, the weight increment is constant set to $0.5 \mathrm{t}$. The last platoon has the opposite arrangement, the platoon starts with the heaviest vehicle at $60 \mathrm{t}$ and the weight is decreased with $0.5 \mathrm{t}$ for each vehicle. The PID controller is purposely set up such that $\left\|G_{i}^{v}(s)\right\|_{\infty}>1$ for a $40 \mathrm{t}$ vehicle, hence the first platoon is not considered string stable. 

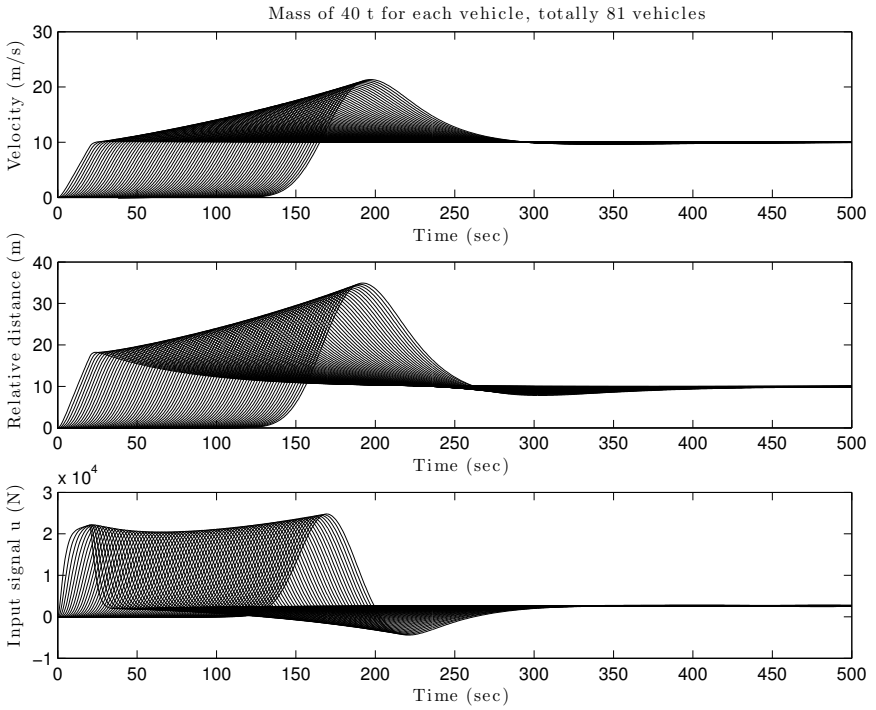

Fig. 2. Simulation results of platoon 1 with identical controllers and identical vehicles of $40 \mathrm{t}$ each. Top plot: The velocity trajectory of the platoon. Middle plot: The relative distance between the vehicles in the platoon. Bottom plot: The input signal from the PID controller for each vehicle.

The simulation of the first platoon consisting of 81 identical vehicles of $40 \mathrm{t}$ each that utilizes identical PID controllers are depicted in Fig. 2. As seen in the figure, for each vehicle in the platoon, the velocity amplifies and this is due to the intentional PID controller setup that does not fulfill the string stability criteria. The relative distance between each vehicle does also amplify throughout the platoon and the input signal from the PID controller is forced to go negative, which can be interpreted as braking. Clearly, this is the platoon behavior that should be avoided.

Figure 3 shows the platoon behavior of the second platoon, where the platoon is arranged to increase for each vehicle starting with $20 \mathrm{t}$ for the lead vehicle and ends with the tail vehicle of $60 \mathrm{t}$. The velocity trajectory of the platoon amplifies similarly to the first platoon. According to the result obtained in (11), this platoon setup should cause the relative distance to amplify for each vehicle, which can be seen in the middle plot of Fig. 3. The maximum relative distance gap is larger than for the first platoon. The larger input signal is mainly due to the heavier vehicles at the rear, but also caused by the larger relative distance.

Lastly, the third platoon with an mass arrangement of decreasing order from $60 \mathrm{t}$ to $20 \mathrm{t}$. The platoon behavior is depicted in Fig. 4 and it can be noted that the behavior differs from the two first platoon arrangement. The velocity does amplify, however it subsides further back in the platoon and the relative distance acts similarly. Considering the adverse PID controller, this platoon arrangement mitigates the slinky effects of the platoon. Hence, this ordering of the platoon improves the robustness of the platoon. If identical controllers are used, the chance for a more robust and a better platoon behavior is when the platoon is arranged in mass decreasing order with the heaviest vehicle first. This could be achieved by utilizing
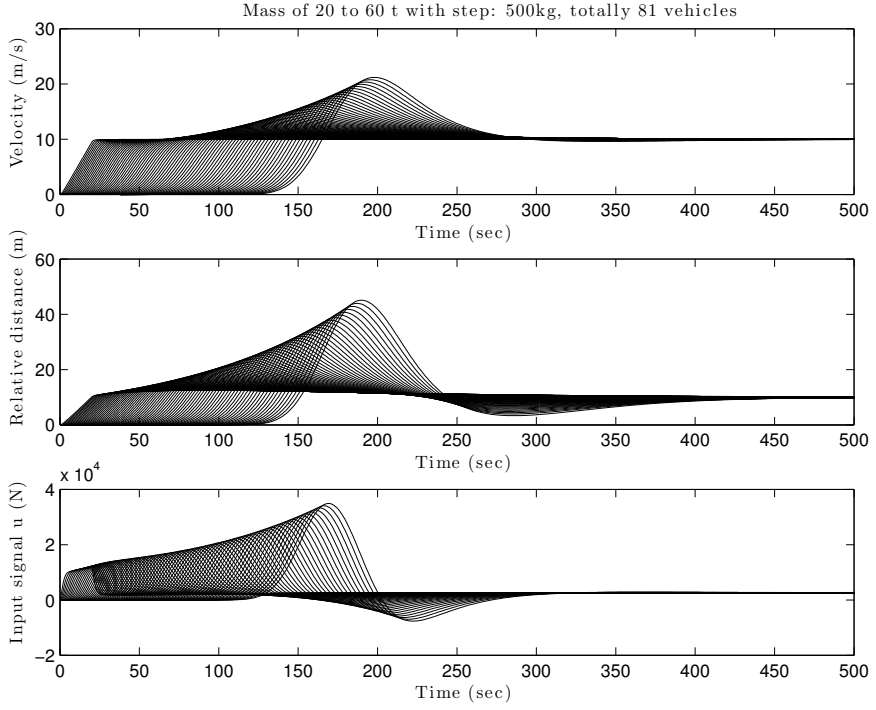

Fig. 3. Simulation results of platoon 2 with identical controllers and an increasing vehicle mass order from $20 \mathrm{t}$ to $60 \mathrm{t}$ at the end. Top plot: The velocity trajectory of the platoon. Middle plot: The relative distance between the vehicles in the platoon. Bottom plot: The input signal from the PID controller for each vehicle.
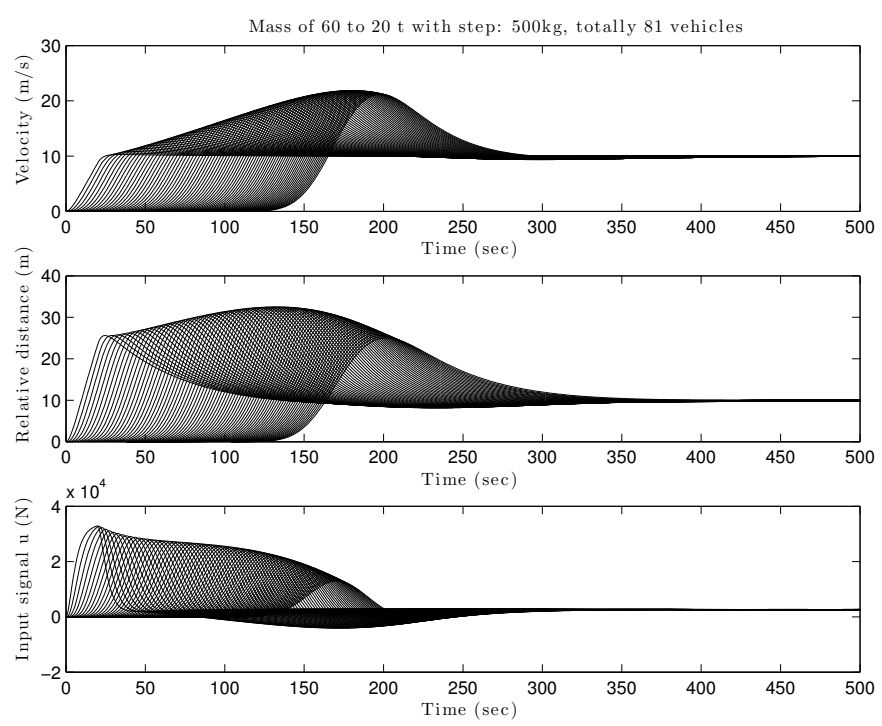

Fig. 4. Simulation results of platoon 3 with identical controllers and a decreasing vehicle mass order from $60 \mathrm{t}$ to $20 \mathrm{t}$ at the end. Top plot: The velocity trajectory of the platoon. Middle plot: The relative distance between the vehicles in the platoon. Bottom plot: The input signal from the PID controller for each vehicle.

$\mathrm{V} 2 \mathrm{~V}$ communication and rearrange the platoon according to the vehicle mass, however in real life application it is difficult to start rearranging a platoon due to other vehicles on the road and the long overtake distances it takes for HDVs.

\section{Control Design}

A proposed solution to avoid any rearrangements of the vehicle order in the platoon is to introduce a PID controller, which takes the mass of the vehicle into account. The derivation of the velocity and relative distance transfer functions with 
the new suggested PID controller are presented in this section, together with a simulation to validate the theory.

\section{A. Mass-dependent PID controller}

The idea is to introduce a mass-dependent factor to the PID controller used earlier, hence every vehicle utilizes the same controller but the output may differ depending on the mass of the vehicle. The proposed controller is expressed as:

$$
\begin{aligned}
\bar{u}_{i}=K_{i}\left[P_{i}\left(d_{i-1,1}-\tau_{d} \dot{x}_{i}\right)\right. & +I_{i} \int d_{i-1, i}-\tau_{d} \dot{x}_{i} \mathrm{~d} t \\
& \left.+D_{i} \dot{d}_{i-1, i}\right]
\end{aligned}
$$

where $K_{i}$ is the mass-dependent variable, $K_{i}\left(m_{i}\right)$.

The velocity and relative distance transfer functions for the mass-dependent PID controller are derived exactly the same as for the regular PID controller, thus the derivation is omitted here. The velocity transfer function, denoted as $\bar{G}_{i}^{v}(s)$, is now described as:

$$
\bar{G}_{i}^{v}(s)=\frac{s^{2} D_{i}+s P_{i}+I_{i}}{s^{3} \frac{m_{i}}{K_{i}}+s^{2}\left(\frac{b_{i}}{K_{i}}+\tau_{d} P_{i}+D_{i}\right)+s\left(P_{i}+\tau_{d} I_{i}\right)+I_{i}}
$$

Similarly, the relative distance transfer function is expressed as:

$$
\bar{G}_{i}^{d}(s)=\frac{\bar{A}_{i}(s)}{\bar{A}_{i-1}(s)} \frac{\bar{B}_{i-1}(s)}{\bar{C}_{i}(s)}
$$

where

$$
\begin{aligned}
& \bar{A}_{i}(s)=s^{2} \frac{m_{i}}{K_{i}}+s\left(\frac{b_{i}}{K_{i}}+\tau_{d} P_{i}\right)+\tau_{d} I_{i} \\
& \bar{B}_{i}(s)=B_{i}(s)=s^{2} D_{i}+s P_{i}+I_{i} \\
& \bar{C}_{i}(s)=s^{3} \frac{m_{i}}{K_{i}}+s^{2}\left(\frac{b_{i}}{K_{i}}+\tau_{d} P_{i}+D_{i}\right)+s\left(P_{i}+\tau_{d} I_{i}\right)+I_{i}
\end{aligned}
$$

This complex transfer function could be simplified with a few assumptions, similarly to $G_{i}^{d}(s)$. With the following assumptions:

$$
\left\{\begin{aligned}
\frac{K_{i-1} P_{i-1}}{m_{i-1}} & \approx \frac{K_{i} P_{i}}{m_{i}} \\
\frac{b_{i-1}}{m_{i-1}} & \approx \frac{b_{i}}{m_{i}} \\
\frac{K_{i-1} I_{i-1}}{m_{i-1}} & \approx \frac{K_{i} I_{i}}{m_{i}}
\end{aligned}\right.
$$

the relative distance transfer function can be approximated to:

$$
\bar{G}_{i}^{d}(s) \approx \frac{m_{i}}{m_{i-1}} \frac{K_{i-1}}{K_{i}} \frac{\bar{B}_{i-1}(s)}{\bar{C}_{i}(s)}
$$

where $\bar{B}_{i-1}(s)$ and $\bar{C}_{i}(s)$ are from(15). It is now possible to obtain a maximum peak equal to 1 or less, $\left\|\bar{G}_{i}^{d}(s)\right\|_{\infty} \leq 1$, by a smart choice of the mass-dependent variable $K_{i}$ regardless the order of the vehicle masses. With the mass-dependent PID controller, the platoon should no longer be dependent on platoon ordering and heterogeneity.
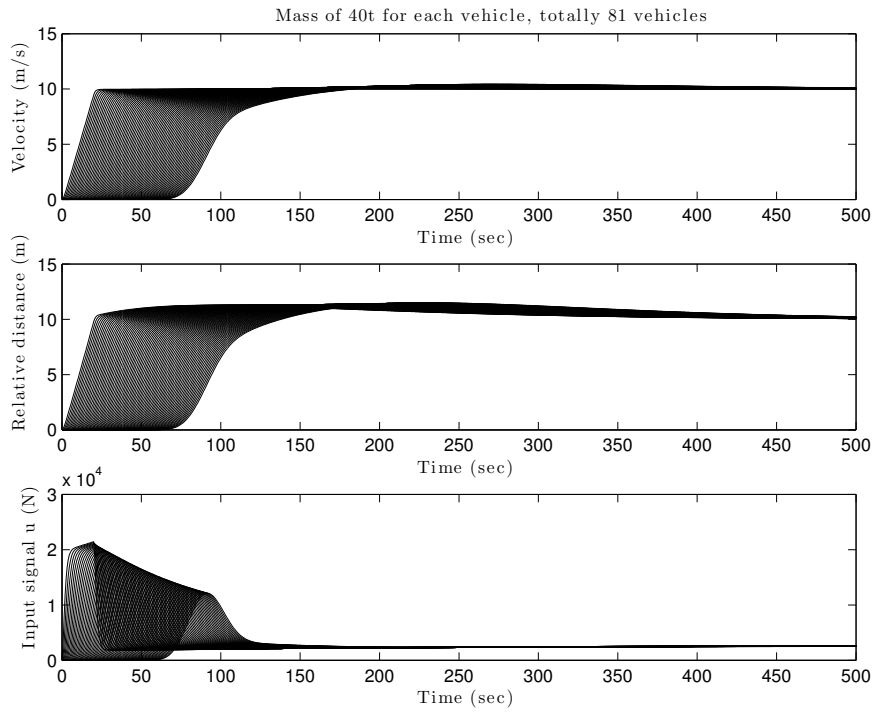

Fig. 5. Simulation results of platoon 1 with identical vehicles of $40 \mathrm{t}$ each and a mass-dependent PID controller. Top plot: The velocity trajectory of the platoon. Middle plot: The relative distance between the vehicles in the platoon. Bottom plot: The input signal from the mass-dependent PID controller for each vehicle.

\section{B. Simulation}

To demonstrate the effect the mass-dependent PID controller has on the platoon behavior compared to a PID controller, the simulation scenario remains with a ramp from $0 \mathrm{~m} / \mathrm{s}$ to $10 \mathrm{~m} / \mathrm{s}$ in $20 \mathrm{~s}$ for the same three platoon arrangements. However, due to the mass-dependent variable $K_{i}$ the velocity transfer function is now $\left\|\bar{G}_{i}^{v}(s)\right\|_{\infty} \leq 1$ for the $40 \mathrm{t}$ vehicle. Thus, the first platoon is considered string stable with the massdependent PID controller in contrast to the PID controller.

The simulation results for the first, second and third platoon are illustrated in Fig. 5, 6 and 7 respectively. Each platoon shows similar platoon behavior in both velocity and relative distance with no slinky effects, thus it is considered a string stable platoon. However, the input signals differ but this is due to the mass of the vehicle that are arranged differently in each platoon. Notice that the controller input never requests for a brake in any of the platoon arrangements. This signifies that a modified PID controller that takes the mass into account can suppress disturbances and make the platoon string stable regardless of the vehicle mass.

\section{SUMMARY \& CONCLUSION}

To study string stability of a HDV platoon, the platoon can be described as a mass-spring-damper system and yet keep the essential behavior of a platoon. When components of a massspring system are identical besides the mass, a damping effect is introduced into the system. This theory is now established on a vehicle platoon to analyze how the masses of the vehicles in the platoon affects the platoon performance. Since all types of automatic control can be described approximately as a PID controller, this study utilizes a PID controller for analysis and identical PID controller is assumed for each vehicle. 

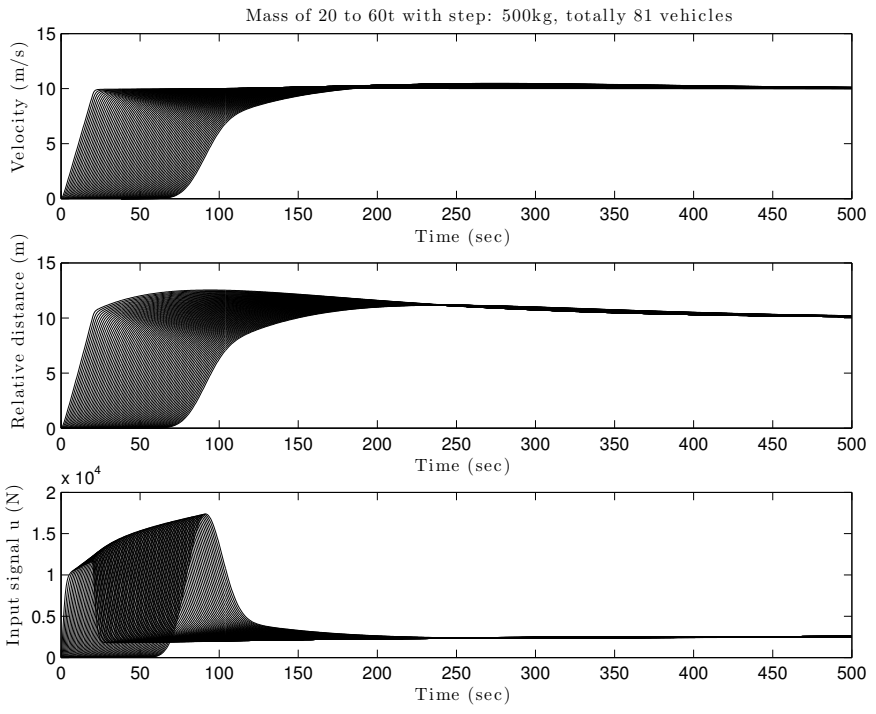

Fig. 6. Simulation results of platoon 2 with a mass decreasing platoon order and a mass-dependent PID controller. Top plot: The velocity trajectory of the platoon. Middle plot: The relative distance between the vehicles in the platoon. Bottom plot: The input signal from the mass-dependent PID controller for each vehicle.

The vehicle only obtains information of the preceding vehicle regarding relative distance and velocity.

The derived transfer function for relative distance indicated a platoon with mass decreasing order with the heaviest vehicle first for a preferable platoon behavior. Intuitively, it seems logical to have a heavier vehicle ahead. A heavier vehicle accelerates and decelerates slower and therefore, the following lighter vehicle can easily follow the heavy vehicle and achieve a shorter braking distance. This is valid with the assumption that all the vehicle properties are identical besides the mass. Although utilizing an adverse PID controller, which is not considered string stable, this platoon arrangement mitigates the slinky effect.

However in a real life scenario, it is inconvenient to start rearranging a platoon due to other vehicles on the highway. Therefore, a proposed solution is to introduce a modified PID controller that takes the vehicle mass into account, in this case, a mass-dependent variable is introduced. Simulations indicated a string stable platoon behavior according to the presented string stable definition, utilizing the modified PID controller, regardless of the vehicle mass and arrangement of the platoon. Also, the input signal from the controller required less force and no braking. This could be comprehended as less torque was produced by the engine, which means a lowered fuel consumption was acquired.

In real life applications a platoon does not often consist of vehicles of the same model from the same manufacturers with identical PID controllers. This makes it more difficult to analyze how the vehicle masses affect string stability. However, through $\mathrm{V} 2 \mathrm{~V}$-communication the $K_{i}$ factor in the controller could be calibrated such that the controller matches with other vehicles by obtaining information of masses and
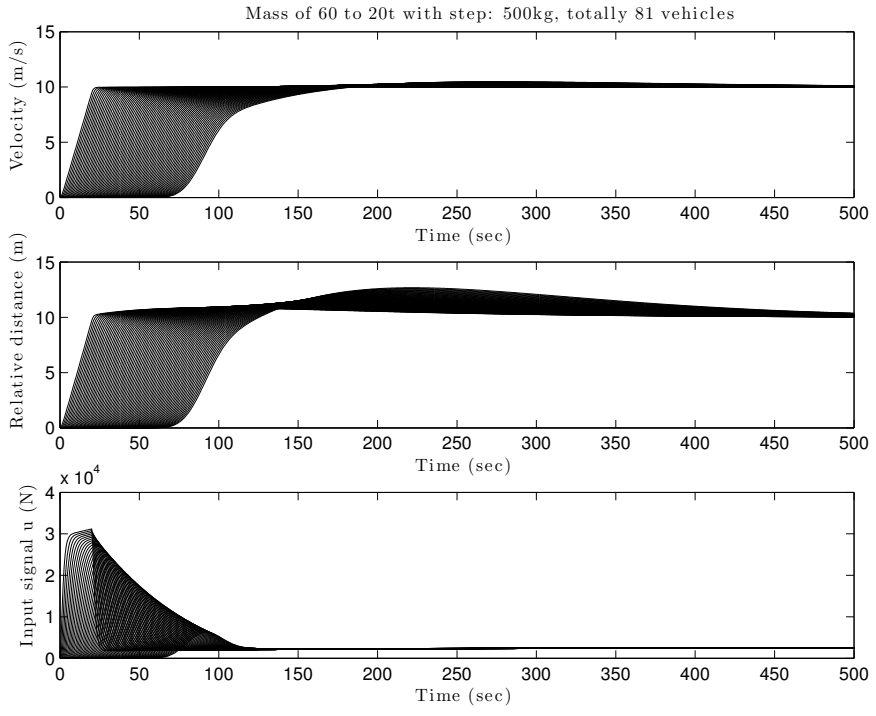

Fig. 7. Simulation results of platoon 3 with with a mass increasing platoon order and a mass-dependent PID controller. Top plot: The velocity trajectory of the platoon. Middle plot: The relative distance between the vehicles in the platoon. Bottom plot: The input signal from the mass-dependent PID controller for each vehicle.

control configuration from other vehicles.

Nevertheless, the mass of a vehicle in a platoon has a significant impact on the platoon behavior. With a small modification to the controller to take its own vehicle mass into account, the platoon behavior can be greatly improved due to the fact that a controller is often optimized for a configuration and does not regard that the mass may change. Therefore, it would be interesting to study this further with a more realistic model. This study used a simplified model of the system, but the real vehicle dynamics are more complicated and consists of nonlinearities with gears, frictions, delays on the system and constraints on the input. In a platoon, the following vehicle experiences an air drag reduction, which is nonlinear with respect to the relative distance. Investigation with a more complex model is left as future work.

\section{REFERENCES}

[1] A. A. Alam, A. Gattami, and K. H. Johansson, "An experimental study on the fuel reduction potential of heavy duty vehicle platooning," in 2010 13th International IEEE Conference on Intelligent Transportation Systems (ITSC), Sep. 2010, ISSN: 2153-0009.

[2] W. Levine and M. Athans, "On the optimal error regulation of a string of moving vehicles," in IEEE Transactions on Automatic Control, vol. AC-11, no.3, 1966.

[3] S. M. Melzer and B. C. Kuo, "Optimal regulation of systems described by a countably infinite number of objects," in Automatica, vol. 7, 1971.

[4] J. L. Willems, "Optimal control of uniform string of moving vehicles," in Ricerche di automatica, vol. 2, 1971.

[5] Y. Zhang, E. B. Kosmatopoulos, P. A. Ioannou, and C. C. Chien, "Using front and back information for tight vehicle following maneuvers," in IEEE Transactions on Vehicular Technology, Jan. 1999, ISSN: 00189545.

[6] M. E. Khatir and E. J. Davison, "Decentralized control of a large platoon of vehicles using non-identical controllers," in Proceedings of the 2004 American Control Conference, 2004, Jun. 2004, ISSN: 0743-1619.

[7] C.-Y. Liang and H. Peng, "Optimal adaptive cruise control with guaranteed string stability," in Proceedings of the 1998 AVEC Conference, 1999. 
[8] H. Hao and P. Barooah, "Control of large 1d networks of double integrator agents: Role of heterogeneity and asymmetry on stability margin," in 201049 th IEEE Conference on Decision and Control (CDC), Dec. 2010, ISSN: 0743-1546.

[9] G. F. Franklin, J. D. Powell, and A. Emami-Naeini, Feedback Control of Dynamic Systems - International Version. Pearson Education, 2008.

[10] D. Swaroop and J. K. Hedrick, "String stability of interconnected systems," in IEEE Transactions on Automatic Control, vol. 41, no.3, mar 1996

[11] Y. Yamamura and Y. Seto, "A study of string-stable ACC using vehicleto-vehicle communication," in 2006 SAE World Congress, ser. 2006-010348, Apr. 2006.

[12] S. Sheikholeslam and C. A. Desoer, "Longitudinal control of a platoon of vehicles with no communication of lead vehicle information: A system level study," in IEEE Tansactions of vehicular technology, vol. 42, no.4, Nov. 1993.

[13] D. Yanakiev and I. Kanellakopoulos, "A simplified framework for string stability analysis in AHS," in In Proceedings of the 13th IFAC World Congress, 1996 